\title{
ANTICONFORMAL AUTOMORPHISMS OF COMPACT RIEMANN SURFACES
}

\author{
ROBERT ZARROW
}

\begin{abstract}
We show that an automorphism of prime order of a compact Riemann surface is embeddable if it is the square of an anticonformal automorphism. Also, every embeddable automorphism of odd order of a compact Riemann surface is the square of an orientation reversing selfhomeomorphism.
\end{abstract}

The results in this paper rely heavily on the work of $R$. Rüedy [3]. It is well known that a smooth surface embedded in $\mathbf{R}^{3}$ inherits a conformal structure from $\mathbf{R}^{3}$. We say a Riemann surface is embeddable if it is conformally equivalent to a smooth surface which is embedded in $\mathbf{R}^{3}$. It has recently been shown that every Riemann surface is embeddable [1], [2]. If $X$ is a Riemann surface then an (anticonformal) automorphism of $X$ is a (anti)conformal homeomorphism of $X$ onto itself. If $X$ has an automorphism $f$ then we say $f$ is embeddable if there is an embedding $d: X \rightarrow \mathbf{R}^{3}$ such that $d f d^{-1}$ is the restriction of a rotation. Now Rüedy [3] has given necessary and sufficient conditions for an automorphism to be embeddable, and for completeness we quote his result here.

Let $\langle f\rangle$ denote the group generated by $f$. Let $F(f)$ denote the set of fixed points of $f$ and $F(\langle f\rangle)=\cup_{j=1}^{r-1} F\left(f^{j}\right)$ where $r$ is the order of $f$. If $p$ is a fixed point then there exists a chart $(D, \phi)$ such that $\phi(p)=0$ and $\phi f \phi^{-1}(z)=z \exp i \alpha$. Now $\alpha=\alpha(f, p)$ is unique up to a multiple of $2 \pi$, independent of the choice of chart. We normalize by requiring $-\pi<\alpha \leqslant \pi$.

THEOREM 1 (RÜEDY). Let $X$ be a compact Riemann surface with an automorphism $f$. Then $f$ is embeddable if and only if:

(1) $F(f)=F(\langle f\rangle)$;

(2) the number of fixed points of $f$ is even;

(3) either $\alpha(f, p)=\pi$ (i.e. $\left.f^{2}=\mathrm{id}\right)$ or $\sum_{p \in F(f)} \alpha(f, p)=0$;

(4) $|\alpha(f, p)|=|\alpha(f, q)|$ for any two fixed points $p$ and $q$.

We apply this result in the following theorem.

THEOREM 2. Let $X$ be a Riemann surface with an automorphism $f$ of prime order $r$. Then $f$ is embeddable if $f=g^{2}$, where $g$ is anticonformal.

Proof. We assume that $f=g^{2}$, where $g$ is anticonformal. If $r=2$ or $f$ has no fixed points then this is immediate, so we assume that $r>2$ and $f$ has fixed points. We now check each of the conditions of Theorem 1 separately. 
(1) If $f^{j}(q)=q$, with $1<j<r$, then (since $\left.(j, r)=1\right)$ there exist integers $k$ and $\ell$ such that $k j+\ell r=1$. Hence $f(q)=f^{k j+\ell r}(q)=f^{k j}(q)=q$. Thus $F(f)=F(\langle f\rangle)$.

(2) It is easy to show that an anticonformal automorphism whose square is not the identity has no fixed points. If $p$ is a fixed point of $f$, then $g(p) \neq p$ and $g(p)$ is also a fixed point of $f$. Thus we may arrange the fixed points of $f$ in pairs and the total number of them is even.

(3) Since $g$ is anticonformal, $\alpha(f, p)=-\alpha(f, g(p))$.

(4) The proof is by induction on the number of fixed points. If there are two fixed points then this is immediate from (3).

Now assume that there are $2 j$ fixed points, $j>1$, and assume that (4) holds when there are $i$ fixed points, where $1 \leqslant i<j$. Let $p$ and $g(p)$ be a pair of fixed points of $f$. Then there exists a pair of open disks $D$ and $D^{\prime}$ in $X$ which have centers at $p$ and $g(p)$, respectively, such that $f$ maps each disk onto itself and such that $g(D)=D^{\prime}$. Notice that $\alpha(p, f)$ is completely determined by the action of $g$ on $\partial D$. Now glue the two boundary curves together by identifying $x \in \partial D$ with $g(x) \in \partial D^{\prime}$. This is again a Riemann surface and $f$ acts on this surface as a holomorphic map. By the induction hypothesis this surface satisfies the conditions of Theorem 1 so that $X$ can be embedded in $\mathbf{R}^{3}$ in such a way that $f$ is the restriction of a rotation. Now let $\gamma$ be the curve obtained by identifying $\partial D$ and $\partial D^{\prime}$. Clearly $f$ maps $\gamma$ onto itself and the angle of rotation of $\gamma$ is the same as $\pm \alpha(q, f)$, where $q \neq p, f(p)$ and $q$ is any one of the remaining fixed points of $f$. Thus $|\alpha(p, f)|=|\alpha(q, f)|$, for all $q \in F(f)$.

As a partial converse we have the following.

THEOREM 3. If $X$ is Riemann surface with an embeddable automorphism $f$ of odd order $r$, then $f$ is the square of an orientation reversing self-homeomorphism.

Proof. It suffices to consider the situation in which $X$ and $f$ are both smooth. We embed $X$ in $\mathbf{R}^{3}$ so that $f$ is the restriction of a rotation through an angle $\alpha$. If $f$ is fixed point free the situation is as in Figure 1. By [3, Lemmal], there exists a simple closed curve $\gamma$ such that $X-\cup_{i=1}^{r} f^{i}(\gamma)$ consists of $r$ components which are permuted by $f$. The closure of each component is a sphere with two boundary components and $n$ handles. Let $\beta=2 \pi / r$ and let $h$ be a rotation through an angle of $\beta$ so that $h$ is a self-homeomorphism of $X$. Thus $h^{m}=f$ for some $m$, where $1 \leqslant m<r$. We may alter the embedding of $X$ in $\mathbf{R}^{3}$, if necessary, so that (1) $X$ is invariant under a rotation $k$ of angle $\beta / 2$ or $\beta(r+1) / 2$, depending on whether $n$ is even or odd, and (2) $X$ is invariant under a vertical reflection $j$ which commutes with $k$. Then $k^{2}=h$ and $f=\left(k^{m} j\right)^{2}$.

In the case in which there are fixed points an analogous proof holds. Here one uses [3, Lemma 2].

REMARK. If, in Theorem 3, we allow $f$ to have even order then only partial results are known. If $f$ has even order greater than 2 , then $f$ is the square of an orientation reversing map provided $n$ is even. (We define $n$ in the proof of Theorem 3.) If $X$ has genus $\ell$ and $f$ is of order 2 with 0 (resp. $2 k+2$, $\ell>k \geqslant 0$ ) fixed points then $f$ is the square of an orientation reversing map if $\ell \equiv 1 \bmod 4($ resp. $\ell \equiv 3 k \bmod 4)$. These facts may be shown by methods 


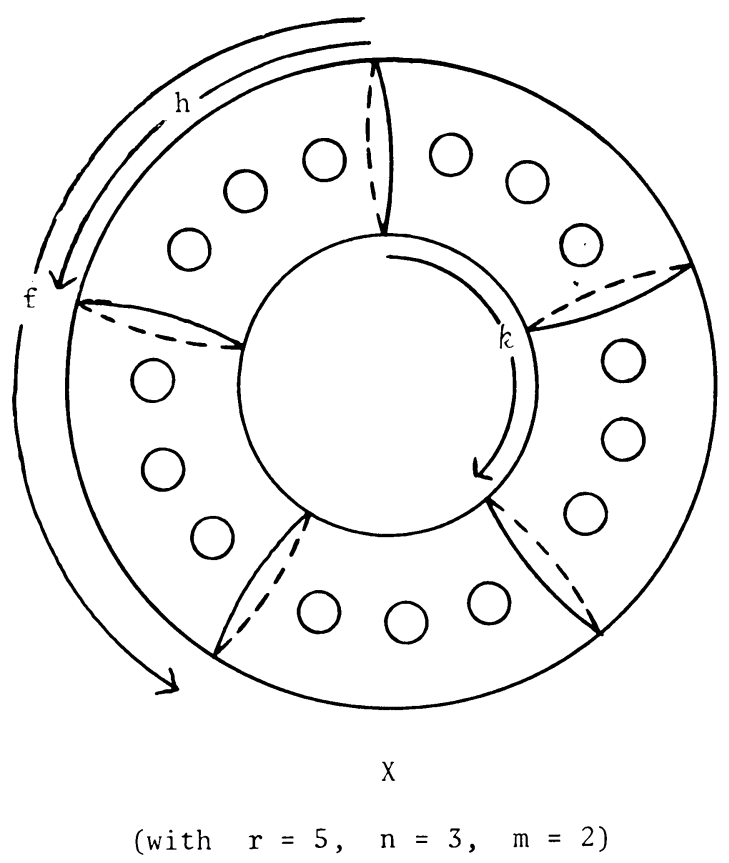

FIGURE 1

similar to those used in proving Theorem 3. Finally, it is shown in [4] that if $f$ is the hyperelliptic involution then it again satisfies the conclusion of Theorem 3 iff the genus of $X$ is even. I do not know if these are the only cases in which an automorphism of order 2 is the square of an orientation reversing map.

\section{REFERENCES}

1. A. M. Garsia, An embedding of closed Riemann surfaces in Euclidean space, Comment. Math. Helv. 35(1961), 93-110. MR23 \# A2890.

2. R. A. Rüedy, Embeddings of open Riemann surfaces, Comment. Math. Helv. 46(1971), 214-225. MR46 \#3781.

3. , Symmetric embeddings of Riemann surfaces, Discontinuous Groups and Riemann Surfaces, Princeton Univ. Press, Princeton, N.J., 1971, pp. 406-418.

4. R. Zarrow, A canonical form for symmetric and skew symmetric extended symplectic modular matrices with applications to Riemann surface theory, Trans. Amer. Math. Soc. 204(1975), 207-227.

Department of Mathematics, Northern Illinois University, DeKalb, Illinois 60115 